\title{
An Efficient Synthesis of Functionalized 1,6-Dienes from Baylis-Hillman Adducts via a Pd-Catalyzed Decarboxylative Protonation Protocol
}

\author{
Bo Ram Park, Ko Hoon Kim, and Jae Nyoung Kim* \\ Department of Chemistry and Institute of Basic Science, Chonnam National University, Gwangju 500-757, Korea \\ *E-mail:kimjn@chonnam.ac.kr \\ Received April 4, 2010, Accepted May 10, 2010
}

Key Words: Palladium, 1,6-Dienes, Baylis-Hillman adducts, Decarboxylative protonation

Functionalized 1,6-dienes is a synthetically important skeleton $^{1,2}$ and has been used for the synthesis of various carbocycles via a ring-closing metathesis (RCM) reaction ${ }^{1 \mathrm{a}}$ and a Pd-catalyzed cyclization/hydrosilylation. ${ }^{\mathrm{b}, \mathrm{c}}$ In addition, symmetric biscinnamic acid derivatives have been used for the synthesis of $C_{2}$-symmetric core units of HIV protease inhibitors, ${ }^{2 \mathrm{a}}$ spiro glutarimides and spiro bisglutarimides, ${ }^{2 \mathrm{~b}}$ and propellano bislactone derivatives. ${ }^{2 \mathrm{c}}$ These functionalized 1,6-dienes are most commonly prepared by diallylation of active methylene compounds with allylic halides under basic conditions. ${ }^{1 \mathrm{e}, 2 \mathrm{~b}, 2 \mathrm{c}} \mathrm{Re}-$ cently, a palladium-catalyzed allylation of active methylene compounds are using widely. ${ }^{1 \mathrm{a}, 3}$ Cinnamyl bromides, derived from the Baylis-Hillman adducts, has also been used in this way for the synthesis of symmetric bis-cinnamic acid derivatives. ${ }^{2 b-e}$

During our recent studies on Pd-catalyzed decarboxylative protonation, allylation, and elimination reactions with modified Baylis-Hillman adducts, ${ }^{4}$ we envisioned that synthetically interesting 1,6-diene derivatives $\mathbf{4}$ could be synthesized, as shown in Scheme 1.

The starting materials 3a-f were prepared by the reactions of various cinnamyl bromides 1a-e, prepared from the corresponding Baylis-Hillman adducts stereoselectively, ${ }^{5}$ and active methylene compounds $\mathbf{2 a}$ or $\mathbf{2} \mathbf{b}$ under the influence of $\mathrm{K}_{2} \mathrm{CO}_{3}$ in $\mathrm{CH}_{3} \mathrm{CN}$ at room temperature. Bis-cinnamylated products

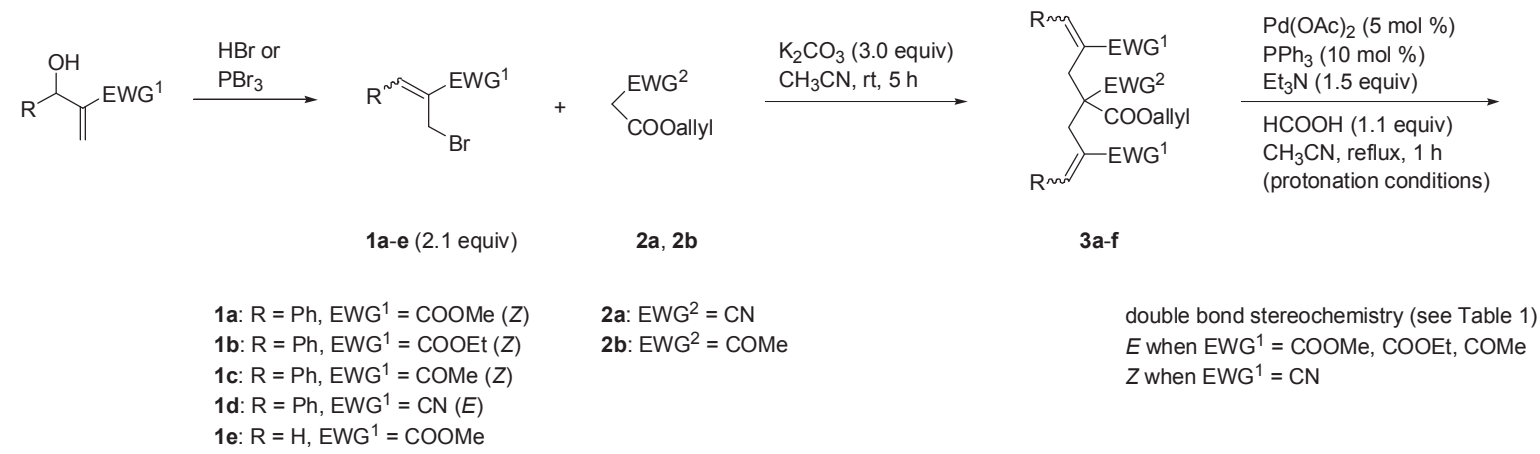

Scheme 1

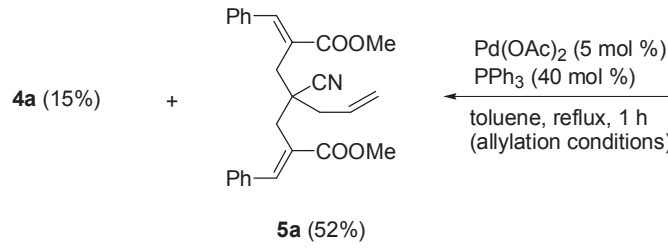

3a $\left.\left.\stackrel{\mathrm{Pd}(0)}{\longrightarrow} \sum_{\mathrm{COOPd}-1}^{\mathrm{CN}}\right)\right\rangle$ double bond stereochemistry (see Table 1) $E$ when $\mathrm{EWG}^{1}=\mathrm{COOMe}$, COOEt, $\mathrm{COMe}$ $Z$ when $E W^{1}=\mathrm{CN}$

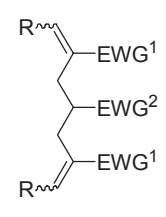

4a-f 
Table 1. Synthesis of $\mathbf{3}$ and Pd-catalyzed decarboxylative protonation to 4

Entry Substrates

${ }^{a}$ Conditions: compound 1 (2.1 mmol), compound $2(1.0 \mathrm{mmol}), \mathrm{K}_{2} \mathrm{CO}_{3}$ (3.0 equiv), $\mathrm{CH}_{3} \mathrm{CN}, \mathrm{rt}, 5 \mathrm{~h} .{ }^{b} \mathrm{Pd}(\mathrm{OAc})_{2}(5 \mathrm{~mol} \%), \mathrm{PPh}_{3}(10 \mathrm{~mol} \%), \mathrm{Et}_{3} \mathrm{~N}$ (1.5 equiv), $\mathrm{HCOOH}$ (1.1 equiv), $\mathrm{CH}_{3} \mathrm{CN}$, reflux, $1 \mathrm{~h}$. ${ }^{c}$ Run at rt, $30 \mathrm{~min}$. ${ }^{d}$ Severe decomposition of $\mathbf{3 f}$ to intractable mixtures and failed to obtain $4 \mathbf{4}$.

3a-f were obtained as the major products (63 - 94\%) along with trace amounts of mono-cinnamylated compounds. The stereochemistry around the double bond is $E$ for $\mathbf{3 a - c}$ and $Z$ for $\mathbf{3 d}$ and 3e. ${ }^{5}$ The next Pd-catalyzed decarboxylative protonation reaction of 3a-f was carried out under the typical conditions involving the use of $\mathrm{Pd}(\mathrm{OAc})_{2}(5 \mathrm{~mol} \%) / \mathrm{PPh}_{3}(10 \mathrm{~mol} \%)$ and $\mathrm{Et}_{3} \mathrm{~N}$ (1.5 equiv)/ $\mathrm{HCOOH}$ (1.1 equiv) in $\mathrm{CH}_{3} \mathrm{CN}$ (reflux, $1 \mathrm{~h}$ ). ${ }^{4,6}$
Desired products 4a-e were isolated in 85 - $96 \%$ yields and the results are summarized in Table 1 . It is interesting to note that the reaction of acetyl derivative $\mathbf{3 e}$ (entry 5 ) showed very fast and clean reaction even at room temperature within short time (30 $\mathrm{min}$ ). As shown in Table 1, the stereochemistry around the double bond is $E$ for $\mathbf{4 a - c}$ and $Z$ for $\mathbf{4 d}$ and $\mathbf{4 e}$. The reaction of methylene derivative $\mathbf{3 f}$ (entry 6 ) did not produce the desired compound $\mathbf{4} \mathbf{f}$ in an appreciable amount, unexpectedly. Severe decomposition of $\mathbf{3} \mathbf{f}$ to intractable side products was observed.

As a next trial, we examined Pd-catalyzed decarboxylative allylation $^{4 \mathrm{~b}, 6}$ and decarboxylation-elimination, ${ }^{4 \mathrm{~b}, \mathrm{e}, 6}$ with compound 3a as a representative example as shown in Scheme 2. Decarboxylative allylation was carried out under the conditions of high loading of $\mathrm{PPh}_{3}\left(\mathrm{Pd} / \mathrm{PPh}_{3}, 1: 8\right),{ }^{4 \mathrm{~b}, 6}$ and allyl derivative 5a was isolated in 52\% yield along with a protonation product 4a $(15 \%){ }^{7}$ Decarboxylation-elimination reaction was performed under the conditions of low loading of $\mathrm{PPh}_{3}\left(\mathrm{Pd} / \mathrm{PPh}_{3}, 1: 1\right)$, as reported in a similar system, ${ }^{4 \mathrm{~b}, \mathrm{e}, 6}$ and desired product $6 \mathbf{6}$ was obtained in $55 \%$ along with $4 \mathbf{a}(32 \%){ }^{7}$ All of the mechanisms for the Pd-catalyzed decarboxylative protonation, allylation, and elimination reactions are summarized in Scheme 2.,6

In summary, we disclosed an efficient synthesis of functionalized 1,6-diene derivatives starting from the Baylis-Hillman adducts via the Pd-catalyzed decarboxylative protonation as the key step.

\section{Experimental Section}

Typical procedure for the preparation of compound $3 a^{4 e}$ A solution of cinnamyl bromide $\mathbf{1 a}(536 \mathrm{mg}, 2.1 \mathrm{mmol})$, allyl cyanoacetate $\mathbf{2 a}(125 \mathrm{mg}, 1.0 \mathrm{mmol})$, and $\mathrm{K}_{2} \mathrm{CO}_{3}(415 \mathrm{mg}, 3.0$ $\mathrm{mmol})$ in $\mathrm{CH}_{3} \mathrm{CN}(4 \mathrm{~mL})$ was stirred at room temperature for $5 \mathrm{~h}$. After the usual aqueous workup and column chromatographic purification process (hexanes/ether, 4:1) compound 3a was obtained as colorless oil, $445 \mathrm{mg}$ (94\%). Other compounds were prepared similarly and the spectroscopic data of $\mathbf{3 a - f}$ are as follows.

Compound 3a: ${ }^{4 \mathrm{e}}$ 94\%; colorless oil; ${ }^{1} \mathrm{H}$ NMR $\left(\mathrm{CDCl}_{3}, 300\right.$ MHz) $\delta 3.15(\mathrm{~s}, 4 \mathrm{H}), 3.77(\mathrm{~s}, 6 \mathrm{H}), 4.49(\mathrm{dt}, J=5.4$ and $1.5 \mathrm{~Hz}$, 2H), 5.22-5.37 (m, 2H), 5.82-5.91 (m, 1H), 7.21-7.25 (m, 4H), $7.31-7.40(\mathrm{~m}, 6 \mathrm{H}), 7.88(\mathrm{~s}, 2 \mathrm{H}) ;{ }^{13} \mathrm{C} \mathrm{NMR}\left(\mathrm{CDCl}_{3}, 75 \mathrm{MHz}\right) \delta$ 33.54, 48.80, 52.09, 67.03, 117.17, 118.49, 127.13, 128.56, $128.71,128.87,130.98,134.54,144.29,167.63,167.99$.

Compound 3b: 81\%; colorless oil; IR (film) 2246, 1748, $1709,1255 \mathrm{~cm}^{-1} ;{ }^{1} \mathrm{H}$ NMR $\left(\mathrm{CDCl}_{3}, 300 \mathrm{MHz}\right) \delta 1.33(\mathrm{t}, J=$ $7.2 \mathrm{~Hz}, 6 \mathrm{H}), 3.14(\mathrm{~s}, 4 \mathrm{H}), 4.24$ (q, $J=7.2 \mathrm{~Hz}, 4 \mathrm{H}), 4.48(\mathrm{dt}, J=$ 5.4 and $1.5 \mathrm{~Hz}, 2 \mathrm{H}), 5.21-5.37(\mathrm{~m}, 2 \mathrm{H}), 5.80-5.91(\mathrm{~m}, 1 \mathrm{H})$, 7.21-7.24 (m, 4H), 7.31-7.40 (m, 6H), 7.87 (s, 2H); ${ }^{13} \mathrm{C} \mathrm{NMR}$ $\left(\mathrm{CDCl}_{3}, 75 \mathrm{MHz}\right) \delta 14.13,33.53,48.84,61.33,67.08,117.29$, 118.47, 127.58, 128.62, 128.71, 128.96, 131.12, 134.76, 144.01, 167.33, 168.15; ESIMS $m / z 524\left(\mathrm{M}^{+}+\mathrm{Na}\right)$. Anal. Calcd for $\mathrm{C}_{30} \mathrm{H}_{31} \mathrm{NO}_{6}$ : C, 71.84; H, 6.23; N, 2.79. Found: C, 71.57; H, $6.51 ; \mathrm{N}, 2.88$.

Compound 3c: 74\%; colorless oil; IR (film) 2246, 1744, $1671,1246 \mathrm{~cm}^{-1} ;{ }^{1} \mathrm{H} \mathrm{NMR}\left(\mathrm{CDCl}_{3}, 300 \mathrm{MHz}\right) \delta 2.40(\mathrm{~s}, 6 \mathrm{H})$, $3.05(\mathrm{~d}, J=13.8 \mathrm{~Hz}, 2 \mathrm{H}), 3.21(\mathrm{~d}, J=13.8 \mathrm{~Hz}, 2 \mathrm{H}), 4.47$ (dt, $J=5.7$ and $1.5 \mathrm{~Hz}, 2 \mathrm{H}), 5.22-5.37(\mathrm{~m}, 2 \mathrm{H}), 5.83-5.96(\mathrm{~m}, 1 \mathrm{H})$, 7.26-7.31 (m, 4H), 7.33-7.43 (m, 6H), $7.66(\mathrm{~s}, 2 \mathrm{H}) ;{ }^{13} \mathrm{C} \mathrm{NMR}$ 
$\left(\mathrm{CDCl}_{3}, 75 \mathrm{MHz}\right) \delta 25.83,32.79,49.19,67.22,117.68,118.67$, 128.71, 128.88, 129.01, 131.38, 134.74, 137.48, 143.76, 168.04, 199.66; ESIMS $m / z 464\left(\mathrm{M}^{+}+\mathrm{Na}\right)$. Anal. Calcd for $\mathrm{C}_{28} \mathrm{H}_{27} \mathrm{NO}_{4}$ : C, 76.17; H, 6.16; N, 3.17. Found: C, 76.46; H, 6.34; N, 3.02.

Compound 3d: ${ }^{4 \mathrm{e}} 70 \%$; white solid, mp $86-88{ }^{\circ} \mathrm{C} ;{ }^{1} \mathrm{H}$ NMR $\left(\mathrm{CDCl}_{3}, 300 \mathrm{MHz}\right) \delta 3.00(\mathrm{~d}, J=13.8 \mathrm{~Hz}, 2 \mathrm{H}), 3.16(\mathrm{~d}, J=$ $13.8 \mathrm{~Hz}, 2 \mathrm{H}), 4.79(\mathrm{dt}, J=6.0$ and $1.2 \mathrm{~Hz}, 2 \mathrm{H}), 5.21-5.41(\mathrm{~m}$, 2H), 5.86-5.99 (m, 1H), 7.24 (s, 2H), 7.38-7.45 (m, 6H), 7.73$7.79(\mathrm{~m}, 4 \mathrm{H}) ;{ }^{13} \mathrm{C} \mathrm{NMR}\left(\mathrm{CDCl}_{3}, 75 \mathrm{MHz}\right) \delta 41.92,50.70,68.24$, $102.41,116.17,117.82,120.36,128.83,129.08,130.16,131.09$, $132.48,150.24,165.81$.

Compound 3e: ${ }^{4 \mathrm{e}} 63 \%$; colorless oil; ${ }^{1} \mathrm{H}$ NMR $\left(\mathrm{CDCl}_{3}, 300\right.$ $\mathrm{MHz}) \delta 2.35$ (s, 3H), $3.14(\mathrm{~d}, J=15.0 \mathrm{~Hz}, 2 \mathrm{H}), 3.21(\mathrm{~d}, J=$ $15.0 \mathrm{~Hz}, 2 \mathrm{H}), 4.73(\mathrm{dt}, J=6.3$ and $1.2 \mathrm{~Hz}, 2 \mathrm{H}), 5.25-5.40(\mathrm{~m}$, 2H), 5.87-6.00 (m, 1H), 7.15 (s, 2H), 7.37-7.44 (m, 6H), 7.67$7.73(\mathrm{~m}, 4 \mathrm{H}) ;{ }^{13} \mathrm{C} \mathrm{NMR}\left(\mathrm{CDCl}_{3}, 75 \mathrm{MHz}\right) \delta 27.61,37.61,63.36$, $67.11,104.27,118.45,120.32,128.90,128.95,130.76,130.78$, 132.91, 149.27, 169.50, 201.85.

Compound 3f: 93\%; colorless oil; IR (film) 2246, 1744, 1724, 1442, 1284, 1216, $1162 \mathrm{~cm}^{-1} ;{ }^{1} \mathrm{H}$ NMR $\left(\mathrm{CDCl}_{3}, 300 \mathrm{MHz}\right) \delta$ $2.90(\mathrm{~d}, J=14.1 \mathrm{~Hz}, 2 \mathrm{H}), 3.05(\mathrm{~d}, J=14.1 \mathrm{~Hz}, 2 \mathrm{H}), 3.77$ (s, $6 \mathrm{H}), 4.62(\mathrm{dt}, J=5.7$ and $1.5 \mathrm{~Hz}, 2 \mathrm{H}), 5.26-5.40(\mathrm{~m}, 2 \mathrm{H}), 5.83-$ $5.96(\mathrm{~m}, 1 \mathrm{H}), 5.86(\mathrm{~s}, 2 \mathrm{H}), 6.43(\mathrm{~s}, 2 \mathrm{H}) ;{ }^{13} \mathrm{C} \mathrm{NMR}\left(\mathrm{CDCl}_{3}, 75\right.$ $\mathrm{MHz}) \delta 37.85,49.63,52.25,67.28,117.44,119.44,130.49$, 130.69, 134.11, 166.55, 167.14; ESIMS $m / z 344\left(\mathrm{M}^{+}+\mathrm{Na}\right)$. Anal. Calcd for $\mathrm{C}_{16} \mathrm{H}_{19} \mathrm{NO}_{6}$ : C, 59.81; H, 5.96; N, 4.36. Found: C, $60.11 ; \mathrm{H}, 6.18 ; \mathrm{N}, 4.29$.

Typical procedure for the synthesis of compound 4a. A solution of $3 \mathbf{a}(237 \mathrm{mg}, 0.5 \mathrm{mmol}), \mathrm{Pd}(\mathrm{OAc})_{2}(6 \mathrm{mg}, 5 \mathrm{~mol} \%)$, $\mathrm{PPh}_{3}(13 \mathrm{mg}, 10 \mathrm{~mol} \%), \mathrm{Et}_{3} \mathrm{~N}(76 \mathrm{mg}, 0.75 \mathrm{mmol}$ ), and $\mathrm{HCOOH}$ (25 mg, $0.55 \mathrm{mmol})$ in $\mathrm{CH}_{3} \mathrm{CN}(1.5 \mathrm{~mL})$ was heated to reflux for $1 \mathrm{~h}$ under $\mathrm{N}_{2}$ atmosphere. After the usual aqueous workup and column chromatographic purification process (hexanes/ EtOAc, 7:1) compound 4a was obtained as colorless oil, 170 $\mathrm{mg}(87 \%)$. Other compounds were prepared similarly and the spectroscopic data of $\mathbf{4 a - e}$ are as follows.

Compound 4a: 87\%; colorless oil; IR (film) 2239, 1711, $1266 \mathrm{~cm}^{-1} ;{ }^{1} \mathrm{H}$ NMR $\left(\mathrm{CDCl}_{3}, 300 \mathrm{MHz}\right) \delta 2.74(\mathrm{dd}, J=13.5$ and $6.9 \mathrm{~Hz}, 2 \mathrm{H}), 2.88(\mathrm{dd}, J=13.5$ and $9.3 \mathrm{~Hz}, 2 \mathrm{H}), 3.39-3.48$ $(\mathrm{m}, 1 \mathrm{H}), 3.83(\mathrm{~s}, 6 \mathrm{H}), 7.22-7.42(\mathrm{~m}, 10 \mathrm{H}), 7.90(\mathrm{~s}, 2 \mathrm{H}) ;{ }^{13} \mathrm{C}$ NMR $\left(\mathrm{CDCl}_{3}, 75 \mathrm{MHz}\right) \delta 29.72,29.79,52.22,120.90,128.24$, 128.65, 128.81, 128.91, 134.71, 143.18, 167.56; ESIMS m/z $412\left(\mathrm{M}^{+}+\mathrm{Na}\right)$. Anal. Calcd for $\mathrm{C}_{24} \mathrm{H}_{23} \mathrm{NO}_{4}$ : C, 74.02; H, 5.95; N, 3.60. Found: C, 74.41; H, 5.79; N, 3.33.

Compound 4b: 85\%; colorless oil; IR (film) 2239, 1705, $1224 \mathrm{~cm}^{-1} ;{ }^{1} \mathrm{H} \mathrm{NMR}\left(\mathrm{CDCl}_{3}, 300 \mathrm{MHz}\right) \delta 1.34(\mathrm{t}, J=7.2 \mathrm{~Hz}$, $6 \mathrm{H}), 2.73(\mathrm{dd}, J=13.8$ and $6.9 \mathrm{~Hz}, 2 \mathrm{H}), 2.87(\mathrm{dd}, J=13.8$ and $9.3 \mathrm{~Hz}, 2 \mathrm{H}), 3.42-3.53(\mathrm{~m}, 1 \mathrm{H}), 4.27$ (q, $J=6.9 \mathrm{~Hz}, 4 \mathrm{H}), 7.28-$ $7.42(\mathrm{~m}, 10 \mathrm{H}), 7.90(\mathrm{~s}, 2 \mathrm{H}) ;{ }^{13} \mathrm{C} \mathrm{NMR}\left(\mathrm{CDCl}_{3}, 75 \mathrm{MHz}\right) \delta$ $14.18,29.77,29.87,61.20,120.95,128.58,128.63,128.72$, 128.91, 134.83, 142.87, 167.09; ESIMS $m / z 440\left(\mathrm{M}^{+}+\mathrm{Na}\right)$. Anal. Calcd for $\mathrm{C}_{26} \mathrm{H}_{27} \mathrm{NO}_{4}$ : C, 74.80; H, 6.52; N, 3.35. Found: C, 74.69; H, 6.89; N, 3.47.

Compound 4c: $85 \%$; white solid, mp $108-110^{\circ} \mathrm{C}$; IR (KBr) 2238, 1666, $1220 \mathrm{~cm}^{-1}$; ${ }^{1} \mathrm{H}$ NMR $\left(\mathrm{CDCl}_{3}, 300 \mathrm{MHz}\right) \delta 2.46(\mathrm{~s}$, $6 \mathrm{H}), 2.69(\mathrm{dd}, J=13.2$ and $6.6 \mathrm{~Hz}, 2 \mathrm{H}), 2.80(\mathrm{dd}, J=13.2$ and $9.6 \mathrm{~Hz}, 2 \mathrm{H}), 3.21-3.32(\mathrm{~m}, 1 \mathrm{H}), 7.38-7.41(\mathrm{~m}, 10 \mathrm{H}), 7.70(\mathrm{~s}$, $2 \mathrm{H}),{ }^{13} \mathrm{C} \mathrm{NMR}\left(\mathrm{CDCl}_{3}, 75 \mathrm{MHz}\right) \delta 25.72,28.57,29.30,121.06$,
128.68, 128.86, 128.92, 134.65, 137.99, 143.40, 199.29; ESIMS $m / z 380\left(\mathrm{M}^{+}+\mathrm{Na}\right)$. Anal. Calcd for $\mathrm{C}_{24} \mathrm{H}_{23} \mathrm{NO}_{2}: \mathrm{C}, 80.64 ; \mathrm{H}$, 6.49; N, 3.92. Found: C, 80.87; H, 6.57; N, 3.79.

Compound 4d: $89 \%$; white solid, mp $99-102{ }^{\circ} \mathrm{C}$; IR (KBr) 2243, 2211, $1449 \mathrm{~cm}^{-1} ;{ }^{1} \mathrm{H}$ NMR $\left(\mathrm{CDCl}_{3}, 300 \mathrm{MHz}\right) \delta 2.82(\mathrm{~d}$, $J=7.2 \mathrm{~Hz}, 4 \mathrm{H}), 3.33-3.42$ (m, 1H), 7.20 (s, 2H), 7.41-7.46 (m, $6 \mathrm{H}), 7.74-7.80(\mathrm{~m}, 4 \mathrm{H}) ;{ }^{13} \mathrm{C} \mathrm{NMR}\left(\mathrm{CDCl}_{3}, 75 \mathrm{MHz}\right) \delta 31.57$, 37.89, 104.96, 118.18, 118.97, 129.30, 129.32, 131.36, 132.95, 148.51; ESIMS m/z $346\left(\mathrm{M}^{+}+\mathrm{Na}\right)$. Anal. Calcd for $\mathrm{C}_{22} \mathrm{H}_{17} \mathrm{~N}_{3}$ : C, 81.71; H, 5.30; N, 12.99. Found: C, 81.45; H, 5.76; N, 12.63.

Compound 4e: 96\%; colorless oil; IR (film) 2209, 1714, $1448 \mathrm{~cm}^{-1} ;{ }^{1} \mathrm{H} \mathrm{NMR}\left(\mathrm{CDCl}_{3}, 300 \mathrm{MHz}\right) \delta 2.29(\mathrm{~s}, 3 \mathrm{H}), 2.56$ (dd, $J=14.1$ and $6.0 \mathrm{~Hz}, 2 \mathrm{H}), 2.80(\mathrm{dd}, J=14.1$ and $8.1 \mathrm{~Hz}$, 2H), 3.38-3.47 (m, 1H), 6.99 (s, 2H), 7.38-7.43 (m, 6H), 7.69$7.73(\mathrm{~m}, 4 \mathrm{H}) ;{ }^{13} \mathrm{C} \mathrm{NMR}\left(\mathrm{CDCl}_{3}, 75 \mathrm{MHz}\right) \delta 31.64,37.70$, 49.51, 107.40, 118.46, 129.05, 129.20, 130.86, 133.34, 146.61, 209.08; ESIMS $m / z 363\left(\mathrm{M}^{+}+\mathrm{Na}\right)$. Anal. Calcd for $\mathrm{C}_{23} \mathrm{H}_{20} \mathrm{~N}_{2} \mathrm{O}$ : C, 81.15; H, 5.92; N, 8.23. Found: C, 80.96; H, 6.04; N, 8.11.

Typical procedure for the synthesis of compound 5a. A solution of $3 \mathbf{a}(237 \mathrm{mg}, 0.5 \mathrm{mmol}), \mathrm{Pd}(\mathrm{OAc})_{2}(6 \mathrm{mg}, 5 \mathrm{~mol} \%)$, $\mathrm{PPh}_{3}(52 \mathrm{mg}, 40 \mathrm{~mol} \%)$ in toluene $(1.5 \mathrm{~mL})$ was heated to reflux for $1 \mathrm{~h}$ under $\mathrm{N}_{2}$ atmosphere. After the usual aqueous workup and column chromatographic purification process (hexanes/ $\mathrm{CH}_{2} \mathrm{Cl}_{2}$ /ether, 15:10:1) compound 5a was obtained as colorless oil, $112 \mathrm{mg}$ (52\%) along with $4 \mathbf{a}$ (29 mg, 15\%).

Compound 5a: 52\%; colorless oil; IR (film) 2233, 1718, $1232 \mathrm{~cm}^{-1}$; ${ }^{1} \mathrm{H} \mathrm{NMR}\left(\mathrm{CDCl}_{3}, 300 \mathrm{MHz}\right) \delta 2.04(\mathrm{~d}, J=7.2 \mathrm{~Hz}$, 2H), $2.76(\mathrm{~d}, J=13.8 \mathrm{~Hz}, 2 \mathrm{H}), 2.85$ (d, $J=13.8 \mathrm{~Hz}, 2 \mathrm{H}), 3.82$ $(\mathrm{s}, 6 \mathrm{H}), 4.96(\mathrm{~d}, J=17.1 \mathrm{~Hz}, 1 \mathrm{H}), 5.07$ (d, $J=9.3 \mathrm{~Hz}, 1 \mathrm{H})$, 5.52-5.65 (m, 1H), 7.22-7.25 (m, 4H), 7.31-7.40 (m, 6H), 7.86 $(\mathrm{s}, 2 \mathrm{H}) ;{ }^{13} \mathrm{C} \mathrm{NMR}\left(\mathrm{CDCl}_{3}, 75 \mathrm{MHz}\right) \delta 32.86,41.94,42.47,52.46$, $120.54,121.37,128.83,128.91,129.01,129.22,132.00,135.48$, 143.60, 168.76; ESIMS $m / z 452\left(\mathrm{M}^{+}+\mathrm{Na}\right)$. Anal. Calcd for $\mathrm{C}_{27} \mathrm{H}_{27} \mathrm{NO}_{4}$ : C, 75.50; H, 6.34; N, 3.26. Found: C, 75.77; H, $6.58 ; \mathrm{N}, 3.41$.

Typical procedure for the synthesis of compound 6a. A solution of $\mathbf{3 a}(237 \mathrm{mg}, 0.5 \mathrm{mmol}), \mathrm{Pd}(\mathrm{OAc})_{2}(11 \mathrm{mg}, 10 \mathrm{~mol} \%$ ), $\mathrm{PPh}_{3}(13 \mathrm{mg}, 10 \mathrm{~mol} \%)$ in toluene $(1.5 \mathrm{~mL})$ was heated to reflux for 30 min under $\mathrm{N}_{2}$ atmosphere. After the usual aqueous workup and column chromatographic purification process (hexanes/ $\mathrm{CHCl}_{3} /$ ether, 20:10:1) compound 6a was obtained as colorless oil, $107 \mathrm{mg}$ (55\%) along with $4 \mathbf{a}(62 \mathrm{mg}, 32 \%)$.

Compound 6a: 55\%; colorless oil; IR (film) 2216, 1717, $1256 \mathrm{~cm}^{-1} ;{ }^{1} \mathrm{H} \mathrm{NMR}\left(\mathrm{CDCl}_{3}, 300 \mathrm{MHz}\right) \delta 3.60(\mathrm{~d}, J=2.1 \mathrm{~Hz}$, $2 \mathrm{H}), 3.87(\mathrm{~s}, 3 \mathrm{H}), 3.88(\mathrm{~s}, 3 \mathrm{H}), 6.78(\mathrm{~m}, 1 \mathrm{H}), 7.21-7.46(\mathrm{~m}$, $10 \mathrm{H}), 7.81(\mathrm{~d}, J=1.2 \mathrm{~Hz}, 1 \mathrm{H}), 7.99(\mathrm{~s}, 1 \mathrm{H}) ;{ }^{13} \mathrm{C} \mathrm{NMR}\left(\mathrm{CDCl}_{3}\right.$, $75 \mathrm{MHz}) \delta 32.67,52.38,52.50,116.69,117.15,126.15,128.62$, 128.84 (2C), 129.18, 129.47, 130.06, 130.54, 134.11, 134.35, 138.59, 143.85, 143.89, 166.37, 167.52; ESIMS $m / z 410\left(\mathrm{M}^{+}+\right.$ Na). Anal. Calcd for $\mathrm{C}_{24} \mathrm{H}_{21} \mathrm{NO}_{4}$ : C, 74.40; H, 5.46; N, 3.62. Found: C, 74.67; H, 5.76; N, 3.59.

Acknowledgments. This research was supported by Basic Science Research Program through the National Research Foundation of Korea (NRF) funded by the Ministry of Education, Science and Technology (2009-0070633). Spectroscopic data were obtained from the Korea Basic Science Institute, Gwangju branch. 


\section{References and Notes}

1. For the synthesis of functionalized 1,6-dienes and their synthetic applications, see: (a) Bassindale, M. J.; Edwards, A. S.; Hamley, P.; Adams, H.; Harrity, J. P. A. Chem. Commun. 2000, 1035-1036. (b) Pei, T.; Widenhoefer, R. A. Org. Lett. 2000, 2, 1469-1471. (c) Widenhoefer, R. A.; Perch, N. S. Org. Lett. 1999, 1, 1103-1105. (d) Nayak, M.; Batra, S. Eur. J. Org. Chem. 2009, 3505-3507. (e) Bhar, S.; Chaudhuri, S. K.; Sahu, S. G.; Panja, C. Tetrahedron 2001, 57, 9011-9016. (f) Schuster, M.; Blechert, S. Angew. Chem. Int. Ed. 1997, 36, 2036-2056. (g) Grubbs, R. H.; Chang, S. Tetrahedron 1998, 54, 4413-4450. (h) Patil, N. T.; Kadota, I.; Shibuya, A.; Gyoung, Y. S.; Yamamoto, Y. Adv. Synth. Catal. 2004, 346, 800804.

2. For the synthesis of bis-cinnamic acid derivatives and their synthetic applications, see: (a) Doi, T.; Hirabayashi, K.; Kokubo, M.; Komagata, T.; Yamamoto, K.; Takahashi, T. J. Org. Chem. 1996, 61, 8360-8361. (b) Basavaiah, D.; Reddy, R. J. Org. Biomol. Chem. 2008, 6, 1034-1039. (c) Basavaiah, D.; Satyanarayana, T. Org. Lett. 2001, 3, 3619-3622. (d) Lee, H. S.; Kim, S. J.; Kim, J. N. Bull. Korean Chem. Soc. 2006, 27, 1063-1066. (e) Kim, S. J.; Lee, H. S.; Kim, J. N. Tetrahedron Lett. 2007, 48, 1069-1072.

3. For the Pd-catalyzed allylations of active methylene compounds, see: (a) Gan, K.-H.; Jhong, C.-J.; Yang, S.-C. Tetrahedron 2008, 64, 1204-1212. (b) Ranu, B. C.; Chattopadhyay, K.; Adak, L. Org. Lett. 2007, 9, 4595-4598. (c) Giambastiani, G.; Poli, G. J. Org. Chem. 1998, 63, 9608-9609.

4. For our recent papers on Pd-catalyzed decarboxylative protonation, allylation, and elimination reactions, see: (a) Gowrisankar, S.; Kim, K. H.; Kim, S. H.; Kim, J. N. Tetrahedron Lett. 2008, 49, 6241-6244. (b) Kim, S. H.; Lee, H. S.; Kim, S. H.; Kim, J. N. Tetrahedron Lett. 2009, 50, 3038-3041. (c) Kim, J. M.; Kim, S. H.; Lee, H. S.; Kim, J. N. Tetrahedron Lett. 2009, 50, 1734-1737. (d) Kim, S. H.; Kim, E. S.; Kim, T. H.; Kim, J. N. Tetrahedron Lett. 2009, 50, 6256-6260. (e) Kim, K. H.; Kim, E. S.; Kim, J. N. Tetrahedron Lett. 2009, 50, 5322-5325. For the Pd-catalyzed reactions of Baylis-Hillman adducts, see: (f) Gowrisankar, S.; Lee,
H. S.; Kim, S. H.; Lee, K. Y.; Kim, J. N. Tetrahedron 2009, 65, 8769-8780.

5. For the synthesis of cinnamyl bromide derivatives in a stereoselective manner from Baylis-Hillman adducts, see: (a) Gowrisankar, S.; Kim, S. H.; Kim, J. N. Bull. Korean Chem. Soc. 2009, 30, 726-728 and further references cited therein. (b) Basavaiah, D.; Reddy, K. R.; Kumaragurubaran, N. Nature Protocols 2007, 2, 2665-2676. (c) Das, B.; Banerjee, J.; Ravindranath, N. Tetrahedron 2004, 60, 8357-8361. (d) Fernandes, L.; Bortoluzzi, A. J.; Sa, M. M. Tetrahedron 2004, 60, 9983-9989. (e) Sa, M. M.; Ramos, M. D.; Fernandes, L. Tetrahedron 2006, 62, 11652-11656. (f) Deng, J.; Hu, X.-P.; Huang, J.-D.; Yu, S.-B.; Wang, D.-Y.; Duan, Z.-C.; Zheng, Z. J. Org. Chem. 2008, 73, 2015-2017. (g) Lee, K. Y.; Lee, Y. J.; Kim, J. N. Bull. Korean Chem. Soc. 2007, 28, 143146. (h) Lee, K. Y.; Park, D. Y.; Kim, J. N. Bull. Korean Chem. Soc. 2006, 27, 1489-1492.

6. For the leading references on Pd-catalyzed decarboxylative protonation, allylation, and elimination reactions, see: (a) Tsuji, J.; Nisar, M.; Shimizu, I. J. Org. Chem. 1985, 50, 3416-3417. (b) Mandai, T.; Imaji, M.; Takada, H.; Kawata, M.; Nokami, J.; Tsuji, J. J. Org. Chem. 1989, 54, 5395-5397. (c) Tsuji, J. Pure Appl. Chem. 1986, 58, 869-878. (d) Marinescu, S. C.; Nishimata, T.; Mohr, J. T.; Stoltz, B. M. Org. Lett. 2008, 10, 1039-1042. (e) Ragoussis, V.; Giannikopoulos, A. Tetrahedron Lett. 2006, 47, 683-687. (f) Tsuji, J.; Yamada, T.; Minami, I.; Yuhara, M.; Nisar, M.; Shimizu, I. J. Org. Chem. 1987, 52, 2988-2995. (g) Waetzig, S. R.; Tunge, J. A. J. Am. Chem. Soc. 2007, 129, 4138-4139. (h) Waetzig, S. R.; Rayabarapu, D. K.; Weaver, J. D.; Tunge, J. A. Angew. Chem. Int. Ed. 2006, 45, 4977-4980. (i) Waetzig, S. R.; Tunge, J. A. J. Am. Chem. Soc. 2007, 129, 14860-14861. (j) You, S.-L.; Dai, L.-X. Angew. Chem. Int. Ed. 2006, 45, 5246-5248. (k) Imao, D.; Itoi, A.; Yamazaki, A.; Shirakura, M.; Ohtoshi, R.; Ogata, K.; Ohmori, Y.; Ohta, T.; Ito, Y. J. Org. Chem. 2007, 72, 1652-1658. (1) Nakamura, M.; Hajra, A.; Endo, K.; Nakamura, E. Angew. Chem. Int. Ed. 2005, 44, 7248-7251. (m) Mohr, J. T.; Behenna, D. C.; Harned, A. M.; Stoltz, B. M. Angew. Chem. Int. Ed. 2005, 44, 6924-6927.

7. Decarboxylative protonation product $\mathbf{4 a}$ must be formed due to trace amounts of moisture in the reaction mixture. ${ }^{4}$ 